\title{
Electrostatic-driven gelation of colloidal
}

\section{nanocrystals}

Taisiia Berestok, ${ }^{\dagger}+$ Pablo Guardia, $,{ }^{\dagger}, *$ Maria Ibáñez, ${ }^{\perp}$ Michaela Meyns, ${ }^{\dagger}$ Massimo Colombo,, Maksym Kovalenko, ${ }^{\perp} \S$ Francesca Peiró ${ }^{\ddagger}, \mid$ and Andreu Cabot ${ }^{\dagger}, \|, *$

${ }^{\dagger}$ Catalonia Institute for Energy Research - IREC, 08930 Sant Adrià de Besòs, Barcelona, Spain

* LENS-MIND, Departament d’Enginyeria Electrònica I Biomèdica, Universitat de Barcelona, 08028, Barcelona, Spain

${ }^{\perp}$ Institute of Inorganic Chemistry, Department of Chemistry and Applied Biosciences, ETH Zürich, CH-8093, Switzerland

" Nanochemistry Department, Istituto Italiano di Tecnologia, via Morego 30, 16130 Genova, Italy

$\S$ EMPA-Swiss Federal Laboratories for Materials Science and Technology, Dübendorf, CH8600, Switzerland

I Institute of Nanoscience and Nanotechnology (In2UB), Universitat de Barcelona, 08028, Barcelona, Spain

॥ ICREA, Pg. Lluís Companys 23, 08010 Barcelona, Spain.

KEYWORDS. Nanocrystals, electrostatic assembly, gel, aerogel, ceria, PbS, gold, CO oxidation, colloid. 
ABSTRACT: The assembly of colloidal nanocrystals (NCs) is a unique strategy to produce porous materials with high crystallinity and unmatched control over structural and chemical parameters. This strategy has been demonstrated mostly for single component nanomaterials. In the present work, we report the gelation of colloidal NC solutions driven by the electrostatic interaction of oppositely charged NCs. A key step for leading this strategy to success is to produce a stable colloidal solution of the positively charged component. We achieved this goal by means of functionalizing NCs with inexpensive and non-toxic amino acids such as glutamine. We demonstrate the combination of positively and negatively charged NCs in the proper concentrations to result in gels with a homogeneous distribution of the two compounds. In this way, porous nanocomposites with virtually any combination can be produced. We illustrate this approach by combining positively charged ceria NCs with negatively charged gold NCs to form $\mathrm{Au}-\mathrm{CeO}_{2}$ gels. These gels were dried from supercritical $\mathrm{CO}_{2}$ to produce highly porous $\mathrm{Au}-\mathrm{CeO}_{2}$ aerogels with specific surface areas of $120 \mathrm{~m}^{2} \mathrm{~g}^{-1}$. The formation of a proper interface is confirmed through the evaluation of the nanocomposite catalytic activity toward the $\mathrm{CO}$ oxidation. We further demonstrate the versatility of this strategy to produce porous metal chalcogenide-metal oxide and metal-metal chalcogenide nanocomposites by the examples of $\mathrm{PbS}-\mathrm{CeO}_{2}$ and $\mathrm{Au}-\mathrm{PbS}$.

\section{INTRODUCTION}

Porous nanocomposites are critical components in the fields of catalysis, chemical sensing and filtering, to mention just a few. Such nanomaterials are generally produced in two steps; first a porous matrix is obtained using sol-gel chemistry methods, and later this base material is impregnated with the second component, which adds the required complementary functionality, 
e.g. a co-catalyst or a light sensitizer. ${ }^{1-3}$ This approach presents important limitations. Sol-gel chemistry routes generally result in amorphous materials that require a thermal sintering to attain proper crystallinity. ${ }^{4-8}$ This sintering step strongly reduces the material surface area and prevents tuning grain size, crystal facets and in some cases crystallographic phase. Additionally, widely used impregnation methods offer a limited control over the distribution and structural/chemical parameters of the impregnated component.

An alternative strategy to produce porous nanomaterials is through the cross-linking of colloidal NCs. ${ }^{5,9,10}$ Colloidal NCs with a plethora of compositions can be currently produced with full control over size, facets and crystal phase. ${ }^{11-20}$ Its assembly into gels and aerogels allows to produce highly porous structures with full control over structural and chemical parameters at the nanometer scale. This strategy has so far been developed mostly to produce single component gels and aerogels, mainly of metals and metal chalcogenides. $5,9,10,21,22$ Few notorious examples exist on the gelation of a colloidal dispersion of different types of NCs into a porous nanocomposite. ${ }^{5,10,23-}$ 26 However, most current approaches to colloidal NC gelation are not well suited for the production of composite gels because they do not include any assembly director that provides the gel with a predefined compositional distribution.

In the present work, we demonstrate the electrostatic interaction between NCs of opposite surface charge to be a very convenient strategy to produce high surface area nanocomposites with virtually any composition. We illustrate this approach using different types of $\mathrm{NCs}\left(\mathrm{Au}, \mathrm{CeO}_{2}\right.$ and $\mathrm{PbS}$ ) having positive and negative surface charge.

\section{EXPERIMENTAL DETAILS}


Materials: Cerium(III) nitrate hexahydrate $\left(\mathrm{Ce}\left(\mathrm{NO}_{3}\right)_{3} \cdot 6 \mathrm{H}_{2} \mathrm{O}, 99 \%\right)$, 1-octadecene (ODE, $\mathrm{C}_{18} \mathrm{H}_{36}$, 90\%), oleylamine (OAm, $\mathrm{C}_{18} \mathrm{H}_{37} \mathrm{~N}, 70 \%$ ), oleic acid (OAc, 90\%), L-glutamine (Gln, $\mathrm{C}_{5} \mathrm{H}_{10} \mathrm{~N}_{2} \mathrm{O}_{3}$, $\geq 99 \%$ ), trifluoroacetic acid (TFA, $\mathrm{C}_{2} \mathrm{HF}_{3} \mathrm{O}_{2}, 99 \%$ ), $\mathrm{N}$-methylformamide (MFA, $\mathrm{C}_{3} \mathrm{H}_{7} \mathrm{NO}, 99.8 \%$ ), lead(II) oxide (99.999\%), tetramethylammonium hydroxide pentahydrate (TMAOH, $\mathrm{C}_{4} \mathrm{H}_{23} \mathrm{NO}_{6}$, $\geq 99 \%$ ), gold(III) chloride trihydrate $\left(\mathrm{HAuCl}_{4} \cdot 3 \mathrm{H}_{2} \mathrm{O}, \geq 99.9 \%\right.$ ), tert-butylamine-borane complex (TBAB, 97\%), 1,2,3,4-tetrahydronaphthalene (tetralin, 99\%), sulfur powder (99.998\%), 2Aminoethanethiol hydrochloride (DEAT, 98\%), and 11-mercaptoundecanoic acid (MUA, 95\%) were purchased from Sigma-Aldrich. Hexane, acetone and methanol were of analytical grade and were purchased from Panreac. All reagents were used as received without further purification. Syntheses were carried out under argon atmosphere using standard Schlenk line techniques. Air and moisture-sensitive chemicals were handled and stored under inert atmosphere.

Synthesis of $\mathrm{CeO}_{2}$ NCs: Ceria NCs were synthesized though the thermal decomposition of cerium precursors in the presence of OAm and ODE as detailed in our previous work. ${ }^{20}$ Briefly in a $25 \mathrm{~mL}$ three neck flask, $0.434 \mathrm{~g}(1 \mathrm{mmol})$ of cerium(III) nitrate hexahydrate were mixed with $2 \mathrm{~mL}$ ( $6 \mathrm{mmol}$ ) of OAm within $4 \mathrm{~mL}$ of ODE under magnetic stirring. After degassing the mixture for 30 minutes at $80^{\circ} \mathrm{C}$, a brown colour solution was formed. The mixture was then heated up under argon flow to $300{ }^{\circ} \mathrm{C}$ at a rate of $15^{\circ} \mathrm{C} / \mathrm{min}$. The mixture was allowed to react at $300{ }^{\circ} \mathrm{C}$ for 60 minutes before cooling down to room temperature. NCs were precipitated and washed with acetone at least four times before suspending them in different organic solvents (hexane, chloroform or toluene) producing stable colloidal dispersions (c.a. $10 \mathrm{mg} / \mathrm{mL}$ ). This sample was labelled $\mathrm{OAm}-\mathrm{CeO}_{2}$.

Synthesis of Au NCs: Au NCs were synthesized following the procedure reported by S. Peng et al. with slight modifications. ${ }^{27}$ In brief, $0.25 \mathrm{mmol}(0.1 \mathrm{~g})$ of gold(III) chloride trihydrate, $10 \mathrm{~mL}$ 
of tetraline and $10 \mathrm{~mL}$ of OAm were loaded into a $50 \mathrm{~mL}$ three neck round bottom flask. The mixture was magnetically stirred under argon flow at room temperature for $30 \mathrm{~min}$. Afterward, a previously prepared solution of $0.5 \mathrm{mmol}$ of $\mathrm{TBAB}, 1 \mathrm{~mL}$ of tetralin and $1 \mathrm{~mL}$ of OAm was injected in the reaction flask. Upon this injection, the colour of the solution turned purple. The mixture was allowed to react for $2 \mathrm{~h}$. NCs were isolated by adding $5 \mathrm{~mL}$ of isopropanol and centrifuged for $5 \mathrm{~min}$ at $7000 \mathrm{rpm}$. The collected precipitate was redispersed in $10 \mathrm{~mL}$ of hexane for later use. This sample was labelled OAm-Au.

Synthesis of PbS NCs: PbS NCs were synthesized using a previously reported procedure. ${ }^{28}$

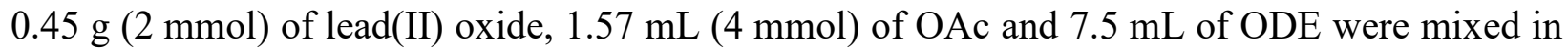
a $25 \mathrm{~mL}$ three neck round bottom flask under magnetic stirring. After $30 \mathrm{~min}$ of degassing at $120^{\circ} \mathrm{C}$, vacuum was switched to Ar atmosphere and the temperature was set to $190{ }^{\circ} \mathrm{C}$. Separately, in a vial, $2 \mathrm{mmol}$ of sulfur powder were dissolved in $3 \mathrm{~mL}$ of OAm by sonication for 10 minutes. This solution was swiftly injected to the reaction flask as soon as this reached $190{ }^{\circ} \mathrm{C}$. The mixture was allowed to react for $5 \mathrm{~min}$ before the heating mantle was removed and the solution cooled down naturally. After washing with acetone at least 3 times, NCs were redissolved in hexane for later use. This sample was labelled OAc-PbS.

Surface modification with GIn: This procedure was inspired by the work of J. De Roo et al. ${ }^{29}$ Ligand exchange processes were held under ambient atmosphere. In a typical procedure, $7 \mathrm{mg}$ of Gln $(0.047 \mathrm{mM})$ was dissolved using mild sonication in $1 \mathrm{~mL}$ MFA followed by the addition of $20 \mu \mathrm{L}$ TFA $(0.2 \mathrm{mM})$. To the obtained mixture, $1 \mathrm{~mL}$ of a $\mathrm{OAm}-\mathrm{CeO}_{2} \mathrm{NC}$ dispersion in hexane $(5 \mathrm{mg} / \mathrm{mL})$ was added, resulting in the formation of a two-phase mixture. The mixture was shaken and sonicated until NCs moved from the upper hexane to the bottom MFA phase, leaving a clear hexane solution on the top. Then the supernatant was removed and a fresh hexane:acetone mixture 
(1:1) was added. The solution was shaken and the NCs were precipitated by centrifugation at $7000 \mathrm{rpm}$ for 10 minutes. This purification procedure was repeated for three times, using MFA to redisperse the NCs at each step. Finally, NCs could be redispersed in polar solvents such as methanol, MFA and water, although colloidal stability in water was moderate. This sample was labeled Gln- $\mathrm{CeO}_{2}$.

Surface modification with MUA: $5 \mathrm{~mL}$ of a $5 \mathrm{mg} / \mathrm{mL}$ dispersion of OAm-Au NCs were mixed with $5 \mathrm{~mL}$ of a $0.02 \mathrm{mM}$ solution of MUA in MFA at $\mathrm{pH}=9$. Level of $\mathrm{pH}$ was adjusted by the addition of TMAOH. The resulting biphasic solution was stirred under inert atmosphere for 30 min. During this process, NCs moved from the upper hexane phase to the bottom MFA phase. Then, the clear upper part was removed and NCs were precipitated by addition of $5 \mathrm{~mL}$ of acetonitrile and centrifugation at $4000 \mathrm{rpm}$ for $5 \mathrm{~min}$. The obtained precipitate was redispersed in $5 \mathrm{~mL}$ of MFA and precipitated one more time with acetonitrile. It should be noted that repeating the washing procedure led to the aggregation of the NCs, but addition of MUA redispersed them back to solution. The sample was labelled MUA-Au. The same process was used with PbS NCs (MUA-PbS).

Surface modification with DEAT: The procedure was inspired by the work reported by Etgar et al. ${ }^{30} 1 \mathrm{~mL}$ of $5 \mathrm{mg} / \mathrm{mL}$ of OAc-PbS NCs dispersed in hexane was added to a solution of $7 \mathrm{mg}$ of DEAT in $1 \mathrm{~mL}$ of MFA. The obtained biphasic mixture was stirred until NCs were transferred from the upper to the bottom phase. Then, the upper part was discarded, and acetone was added to the remaining MFA solution containing the NCs. Then, PbS NCs were precipitated and subsequently washed at least two more times by the addition of MFA as a solvent and acetone and acetonitrile as antisolvent. Finally, NCs were redispersed in MFA for later use. PbS NCs obtained after surface modification with DEAT were labeled as DEAT-PbS. 
NCs assembly: $2.5 \mathrm{~mL}$ of a $5 \mathrm{mg} / \mathrm{mL}$ MUA-Au NC solution in MFA was injected into $2.5 \mathrm{~mL}$ of a $5 \mathrm{mg} / \mathrm{mL}$ Gln-CeO 2 NCs solution in MFA. Immediately after mixing, NCs started to aggregate to form a gel. The solution was left undisturbed for $30 \mathrm{~min}$, until the supernatant became transparent, which indicated the completion of the gelation by the consumption of all the NCs. MFA was then carefully replaced with acetone (approximately $5 \mathrm{~mL}$ ). Solvent exchange with fresh acetone was repeated at least 6 times to ensure complete removal of MFA. The same procedure was followed to prepare $\mathrm{PbS}-\mathrm{CeO}_{2}$ and $\mathrm{Au}-\mathrm{PbS}$ NC-based gels mixing the proper types of NCs.

Aerogel formation: The gel soaked in acetone was loaded into a custom designed supercritical point drier (SPD). The chamber was sealed and it was slowly filled with liquid $\mathrm{CO}_{2}$ until the pressure reached 73 bars. To ensure that $\mathrm{CO}_{2}$ was in liquid phase, temperature was maintained at $17-18^{\circ} \mathrm{C}$ by using a Peltier element and the pressure level was set higher than 73 bars. After storing the sample undisturbed overnight, the liquid $\mathrm{CO}_{2}$ inside of the chamber was half-drained and flushed with fresh one. This exchange was repeated at least 6 times in one hour intervals in order to completely replace acetone with liquid $\mathrm{CO}_{2}$. After the solvent exchange was completed, the chamber at 73 bars was heated up to $39^{\circ} \mathrm{C}$ using a heating mantle which drove the transition of the $\mathrm{CO}_{2}$ from the liquid to the supercritical phase. The sample was kept under these conditions $\left(39^{\circ} \mathrm{C}\right.$ and 90 bars) for $1 \mathrm{~h}$, followed by a slow release of the pressure while keeping the temperature constant.

Structural and chemical characterization: Transmission electron microscopy (TEM) characterization was carried out using a ZEISS LIBRA 120, operating at $120 \mathrm{kV}$. Dark field and high resolution TEM (HRTEM) studies were performed in a JEOL 2200FS TEM operating at an accelerating voltage of $200 \mathrm{kV}$. Samples were prepared by drop casting a diluted solution of the NCs onto a carbon coated cooper grid (200 mesh). Images were analyzed with the Gatan Digital 
Micrograph software. FTIR spectroscopy investigations were carried out using a PerkinElmer FTIR 2000 spectrophotometer. Spectra were recorded form $500 \mathrm{~cm}^{-1}$ to $4000 \mathrm{~cm}^{-1}$. Dynamic light scattering (DLS) and zeta potential measurements were performed using a Zeta Sizer (Malvern Instruments) equipped with a $4.0 \mathrm{~mW} \mathrm{HeNe}$ laser operating at $633 \mathrm{~nm}$ and an avalanche photodiode detector. Specific surface area measurements were carried out by nitrogen physisorption at $77 \mathrm{~K}$ in a Quantachrome equipment, model Autosorb iQ. Prior to measurements, samples were annealed at $400{ }^{\circ} \mathrm{C}$ for $4 \mathrm{~h}$ under air atmosphere using a heating ramp of $2{ }^{\circ} \mathrm{C} / \mathrm{min}$. Then the samples were degassed for 6 hours at $250{ }^{\circ} \mathrm{C}$ under vacuum to eliminate any weakly adsorbed species. The specific surface areas were calculated using the multi-point BET (BrunauerEmmett-Teller) model, considering 11 equally spaced points in the $\mathrm{P} / \mathrm{P}^{0}$ range from 0.05 to 0.30 .

Catalytic test: The catalytic activity toward $\mathrm{CO}$ oxidation of the $\mathrm{CeO}_{2}$ and $\mathrm{Au}-\mathrm{CeO}_{2}$ aerogels was measured using a micro reactor system coupled with a NDIR analyzer that allowed the continuous detection of $\mathrm{CO}$ and $\mathrm{CO}_{2}$ concentrations at the reactor outlet. Typically, $15 \mathrm{mg}$ of the aerogel was loaded into a quartz reactor (internal diameter $=6 \mathrm{~mm}$ ). The feed gas was a mixture of $1 \% \mathrm{v} / \mathrm{v}$ $\mathrm{CO}$ and $10 \% \mathrm{v} / \mathrm{v} \mathrm{O}_{2}$ balanced with $\mathrm{He}$. The flow rate was set to $40 \mathrm{ncc} / \mathrm{min}$. The catalysts were tested directly after synthesis, without any pretreatment. For one test cycle, the reactor was heated from room temperature to $400{ }^{\circ} \mathrm{C}$ with a heating rate of $5{ }^{\circ} \mathrm{C} / \mathrm{min}$ and kept at $400{ }^{\circ} \mathrm{C}$ for $30 \mathrm{~min}$, then cooled down to RT. To ensure that the obtained activity results were reliable, the test cycle for each catalyst was repeated 4 times. Transient activity data were collected during the test, and the $\mathrm{CO}$ conversion was defined as the percentage of $\mathrm{CO}$ feed that had reacted.

\section{RESULTS AND DISCUSSION}

Quasi-spherical $\mathrm{CeO}_{2} \mathrm{NCs}$ with an average diameter of $7 \pm 1 \mathrm{~nm}$ were produced following our previously reported procedure. ${ }^{20} \mathrm{CeO}_{2} \mathrm{NCs}$ were colloidally stable in non-polar organic solvents 
due to the presence of OAm on their surface. Gold NCs of $4 \pm 1 \mathrm{~nm}$ were produced following the procedure reported by Peng et al. also using OAm as surface ligand. ${ }^{27}$ Figure 1 shows representative TEM micrographs of the $\mathrm{OAm}-\mathrm{CeO}_{2}$ and $\mathrm{OAm}-\mathrm{Au}$ NCs used in the present work and their hydrodynamic size distribution obtained from DLS measurements in hexane.
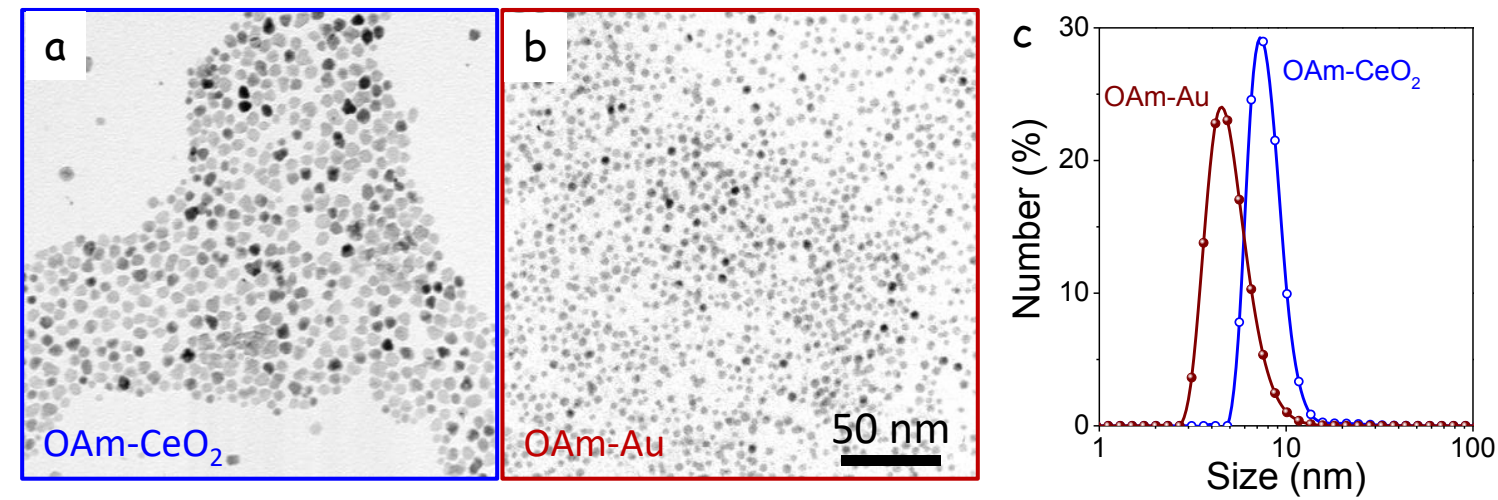

Figure 1. a) TEM image of OAm-CeO 2 NCs. b) TEM image of OAm-Au NCs. c) DLS spectra of $\mathrm{OAm}-\mathrm{CeO}_{2}$ and OAm-Au NCs.

A first key step toward electrostatic assembly is the introduction of opposite charges at the surface of the different NCs to be assembled. While a variety of methods is available to create a negatively charged ligand surface, very few are reported for positive electrostatic stabilization. ${ }^{31}$ Additionally, in most reported procedures the reagents used to introduce a positive surface charge can be harmful and need to be handled with care and/or they are excessively expensive for real application. ${ }^{32,33}$ To introduce a positive charge at the surface of $\mathrm{CeO}_{2} \mathrm{NCs}$, we replaced OAm with safe and inexpensive amino acids (Figure 2). ${ }^{29,34}$ Briefly, an MFA solution of Gln and TFA was mixed with a hexane solution of $\mathrm{CeO}_{2} \mathrm{NCs}$. Through moderate stirring, $\mathrm{CeO}_{2} \mathrm{NCs}$ moved from the hexane phase to the MFA. Then, the hexane phase was discarded and NCs were purified by multiple precipitation and redispersion steps (see details in the experimental section). In the presence of TFA, the amino acid bonded to the metal atoms on the NCs surface through the 
carboxylate group, leaving a protonated amino group exposed to the medium, thus providing a positive charge to the NC. A positive zeta potential peak at $+26 \mathrm{mV}$ confirmed the positive surface charge of the $\mathrm{Gln}-\mathrm{CeO}_{2} \mathrm{NCs}$ (Figure 2f). The presence of $\mathrm{Gln}$ at the $\mathrm{CeO}_{2} \mathrm{NC}$ surface was further proved by peaks at around $1538 \mathrm{~cm}^{-1}$ and $1650 \mathrm{~cm}^{-1}$ in the FTIR spectrum, which were ascribed to $\mathrm{N}-\mathrm{C}=\mathrm{O}$ and $\mathrm{NH}_{2}$ vibration modes of the second amine group of the Gln molecules (Figure 2d). ${ }^{35,36}$

To introduce a negative charge at the surface of Au NCs, OAm was replaced by MUA. The exchange from OAm to MUA was performed via a two-phase ligand exchange procedure by mixing OAm-Au NCs in hexane with a MUA solution in MFA. After agitation, MUA-Au NCs moved to the MFA phase (see experimental section for details). In the presence of a strong base, TMAOH, the thiol (-SH) and carboxyl (-COOH) groups of MUA were deprotonated, which facilitated the bonding of MUA to the NCs surface. For the particular case of Au, the thiol group bonded to the surface due to its strong affinity with $\mathrm{Au}$, thus leaving the acid group exposed to the media. In these conditions, the acid group provided the $\mathrm{NC}$ with a negative surface charge. ${ }^{37,38} \mathrm{The}$ FTIR spectrum of MUA-Au NCs showed absorption bands in the 2820$2946 \mathrm{~cm}^{-1}$ region, which were attributed to C-H stretching vibration from the carboxylic acid of MUA ligands ${ }^{39}$ Additionally, the disappearance of the broad peak at around $2600 \mathrm{~cm}^{-1}$ associated to S-H stretching confirmed the ligand attachment to the NCs surface through deprotonated thiol head group..$^{39,40}$ The peaks at $1571 \mathrm{~cm}^{-1}$ and $1401 \mathrm{~cm}^{-1}$ associated to carboxylate vibrations suggested the presence of the carboxyl salt on the NCs surface. ${ }^{40}$ A negative zeta potential peak at $-50 \mathrm{mV}$ further demonstrated the attachment of MUA to Au NCs and the negative surface charge (Figure 2f). 

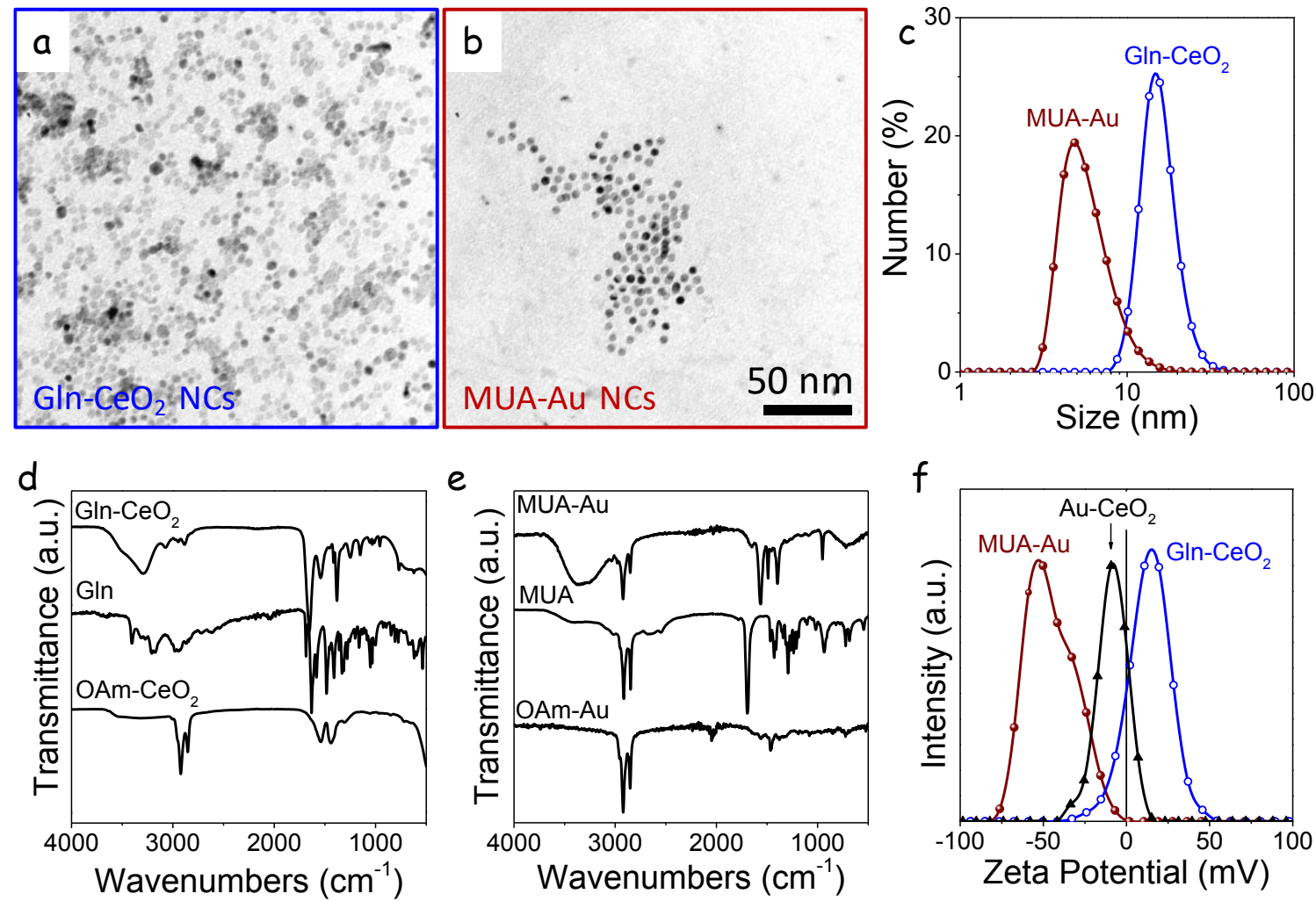

Figure 2. a) TEM image of Gln- $\mathrm{CeO}_{2}$ NCs. b) TEM image of MUA-Au NCs. c) DLS size distribution of Gln- $\mathrm{CeO}_{2}$ and MUA-Au NCs. d) FTIR spectra of $\mathrm{OAm}-\mathrm{CeO}_{2} \mathrm{NCs}$, Gln and Gln$\mathrm{CeO}_{2}$ NCs. e) FTIR spectra of OAm-Au NCs, MUA and MUA-Au NCs. f) Zeta potential curves of Gln- $-\mathrm{CeO}_{2} \mathrm{NCs}$, MUA-Au NCs and the gel formed after mixing Gln-CeO $\mathrm{NCs}_{2}$ and $\mathrm{MUA}-\mathrm{Au}$ NCs.

Both, Gln-CeO $\mathrm{C}_{2}$ and MUA-Au NCs were colloidally stable in MFA. However, when combining MFA solutions of Gln- $\mathrm{CeO}_{2} \mathrm{NCs}$ and MUA-Au NCs, a random aggregation of the NCs into a gel took place. The gelation was moderately fast, reaching completion after about $30 \mathrm{~min}$. For this process to be successful, the high dielectric constant of MFA $(\varepsilon=171)$ was very convenient, both to properly stabilize each type of charged colloidal $\mathrm{NC}$ in solution and to allow gel formation upon their mixing. The NC-based gel was dried from super-critical $\mathrm{CO}_{2}$ to maintain the porous structure (see experimental section for details). After drying, a very low density material with a dark garnet 
color was obtained. Figure 3 shows representative TEM, SEM and optical images of the gel and aerogel produced following this process, using a 4:1 mass ratio of $\mathrm{Gln}-\mathrm{CeO}_{2}$ to $\mathrm{MUA}-\mathrm{Au} \mathrm{NCs}$, which corresponds to a $\mathrm{CeO}_{2}$ : $\mathrm{Au} \mathrm{NC}$ ratio of ca. 2:1. A highly porous structure with large open pores and voids consistent with the nature of an aerogel was evident from the SEM image of the dried gel (Figure 3b). Figure 3c shows the adsorption-desorption isotherm of the same $\mathrm{Au}-\mathrm{CeO}_{2}$ aerogel. From these curves, a BET surface area of $120 \pm 10 \mathrm{~m}^{2} / \mathrm{g}$ was calculated, which is a very high value taking into account the high density of the two compounds, especially Au. Figure 4 displays bright field and dark field TEM micrographs and HRTEM micrographs of the Au-CeO 2 gel formed, showing the homogeneous distribution of the two compounds.
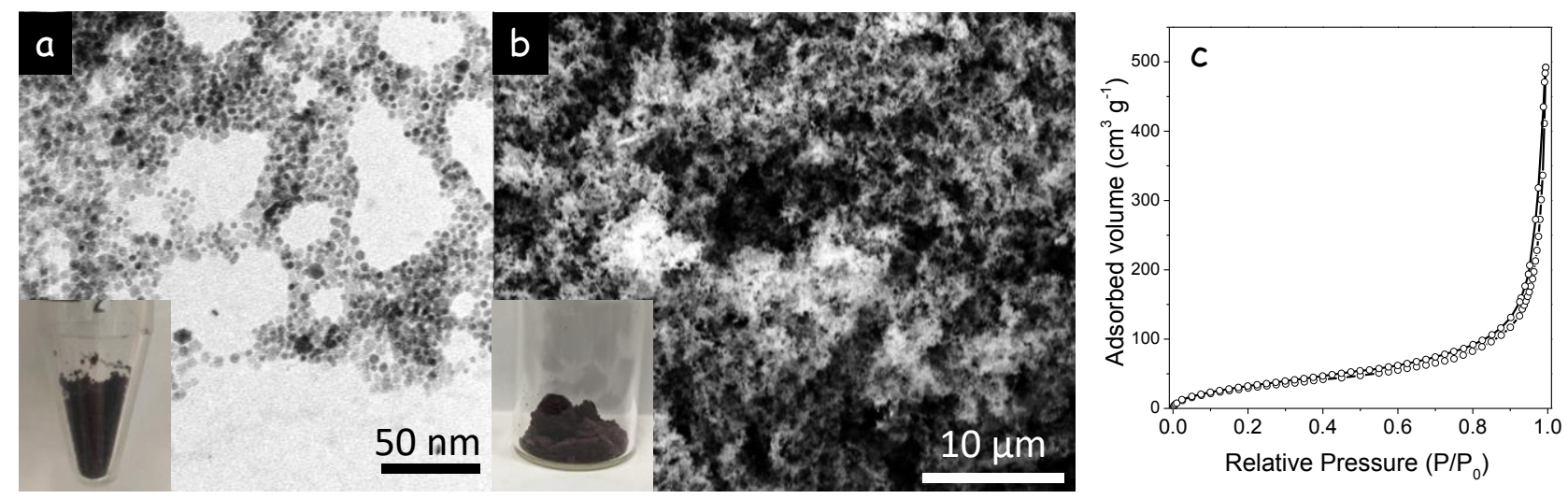

Figure 3. a-b) TEM (a) and SEM (b) image of the Au- $\mathrm{CeO}_{2} \mathrm{NCs}$ gel and aerogel, respectively, obtained by mixing $4 \mathrm{ml}$ of a $5 \mathrm{mg} / \mathrm{mL}$ MFA solution of $\mathrm{Gln}-\mathrm{CeO}_{2} \mathrm{NCs}$ with $1 \mathrm{ml}$ of a $5 \mathrm{mg} / \mathrm{mL}$ MFA solution of MUA-Au NCs. Optical photographs of the gel and the aerogel are shown as insets in $\mathrm{a}$ and $\mathrm{b}$ respectively. c) Adsorption-desorption isotherm measured from the $\mathrm{Au}-\mathrm{CeO}_{2} \mathrm{NCs}$ aerogel. 


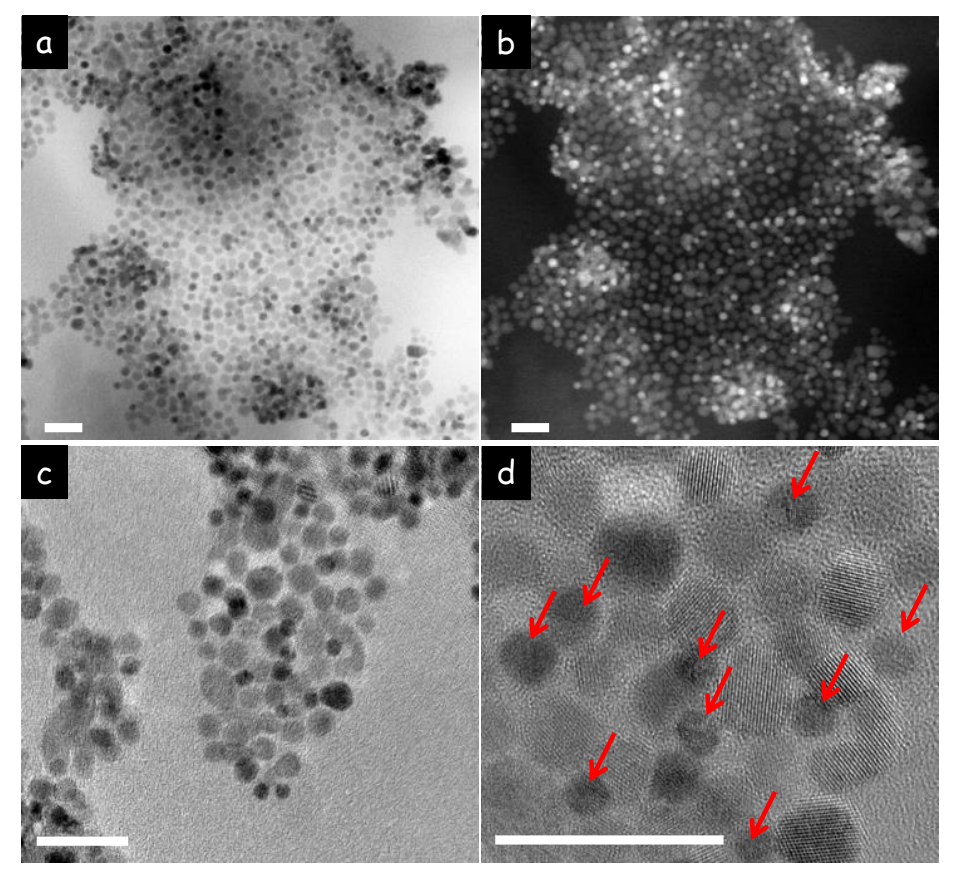

Figure 4. Bright field (a), dark field (b) and high resolution (c,d) TEM micrographs of the 4:1 $\mathrm{CeO}_{2}$ :Au mass ratio gel. Gold NCs appear brighter in the dark field TEM micrograph. Gold NCs are pointed out with red arrows in the HRTEM micrograph in (d). All scale bars correspond to $20 \mathrm{~nm}$.

NCs gelation was driven via electrostatic interaction of oppositely charged NCs (Figure 5a). Gelation occurred within solutions containing a relatively wide range of $\mathrm{CeO}_{2}$ and $\mathrm{Au} \mathrm{NC}$ ratios and concentrations. To determine the $\mathrm{CeO}_{2}$ and $\mathrm{Au} \mathrm{NC}$ ratio required for gel formation, small amounts of a $\mathrm{Au} \mathrm{NC}$ solution were stepwise added into a colloidal solution of $\mathrm{CeO}_{2} \mathrm{NCs}$ until gelation occurred. Following this procedure, we observed the gelation to take place already at a $\mathrm{Au}: \mathrm{CeO}_{2} \mathrm{NC}$ mass ratio of 3:25 (Figure 5b), equivalent to a ca. 6:25 NC ratio. When stepwise adding a $\mathrm{CeO}_{2} \mathrm{NC}$ solution into colloidal $\mathrm{Au} \mathrm{NCs}$, gelation started at a $\mathrm{CeO}_{2}$ : $\mathrm{Au}$ mass ratio of 10:25, equivalent to a $\mathrm{NC}$ ratio of ca. 5:25.

In terms of total $\mathrm{NC}$ concentration (Figure 5c), when using very high $\mathrm{NC}$ concentrations (50 $\mathrm{mg} / \mathrm{mL}$, on the limit of their colloidal stability) a very fast aggregation took place, resulting in 
a NC precipitate rather than a gel. On the other extreme, when the concentration of the NCs was very low $(0.005 \mathrm{mg} / \mathrm{mL})$ we did not observe any $\mathrm{NC}$ aggregation for days due to an insufficient interaction of the NCs in solution (Figure 5). At moderate concentrations, $0.05-5 \mathrm{mg} / \mathrm{mL}$, the solution started to gelate right after the oppositely charged NCs were combined. Within this range, we observed that the lower the $\mathrm{NC}$ concentration the larger the voluminosity of the formed gel (Figure $5 \mathrm{~b}$ ). Note that in Figure $5 \mathrm{~b}$ the gel obtained from combining $200 \mu \mathrm{L}$ of a $0.05 \mathrm{mg} / \mathrm{mL}$ solution of $\mathrm{Gln}-\mathrm{CeO}_{2}$ and $50 \mu \mathrm{L}$ of a $0.05 \mathrm{mg} / \mathrm{mL}$ solution of MUA-Au had approximately the same volume as that obtained from combining the same amounts of $0.5 \mathrm{mg} / \mathrm{mL}$ and $5 \mathrm{mg} / \mathrm{mL} \mathrm{NC}$ solutions, which contained one and two orders of magnitude larger quantities of NCs, respectively.

$a$

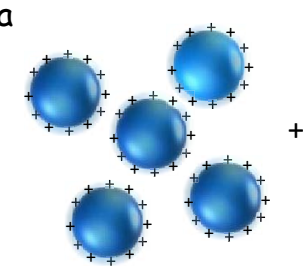

b

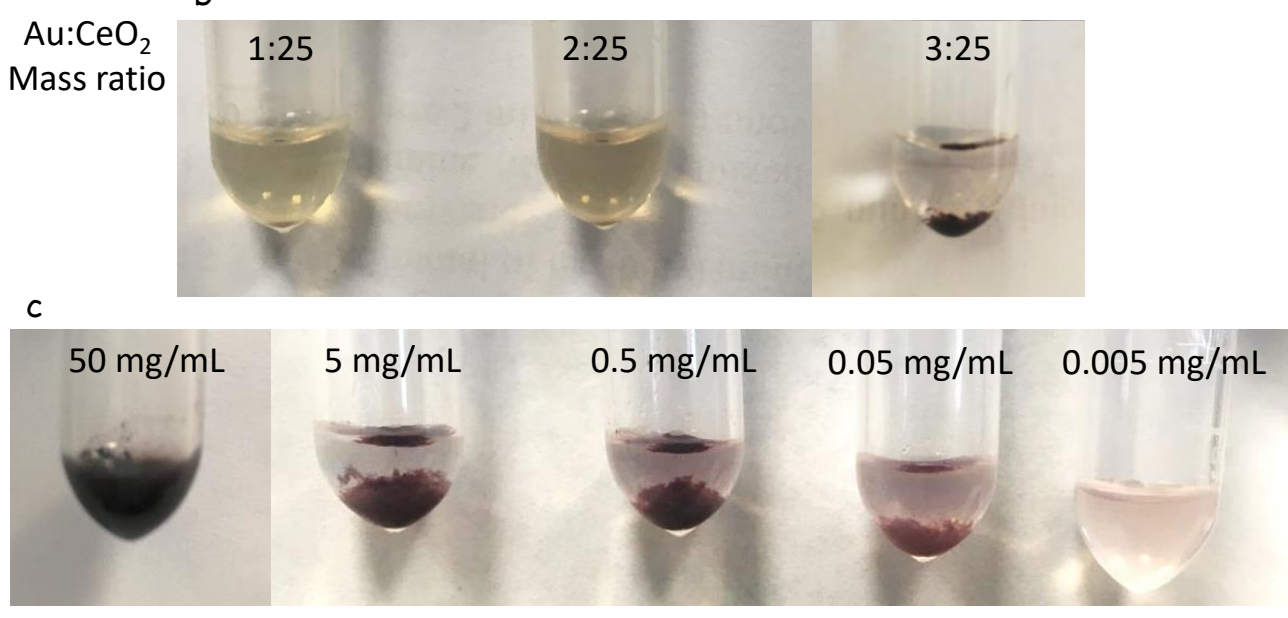

Figure 5. a) Schematic representation of the gelation mechanism based on electrostatic interaction between oppositely charged NCs. b) Optical photographs of a colloidal solution of $\mathrm{CeO}_{2} \mathrm{NCs}$ where different amounts of $\mathrm{Au}$ NCs were stepwise added to reach $\mathrm{Au}: \mathrm{CeO}_{2}$ mass ratios of 1:25; $2: 25 ; 3: 25$, as noted in each photograph. At a concentration of $3: 25$ gelation was observed. c) 
Optical photographs of Au-CeO 2 NCs gels obtained by combining $2 \mathrm{~mL}$ of a $\mathrm{Gln}-\mathrm{CeO}_{2}$ solution and $0.5 \mathrm{~mL}$ of a MUA-Au solution having increasingly higher (from right to left) concentrations $(0.05,0.5,5,50 \mathrm{mg} / \mathrm{mL})$. Thus an order of magnitude in the amount of NCs exists between adjacent samples. Note that the $\mathrm{CeO}_{2}: \mathrm{Au}$ mass ratio was fixed at 4:1. The $\mathrm{Au}-\mathrm{CeO}_{2}$ nanocomposite obtained from the $50 \mathrm{mg} / \mathrm{mL}$ solutions did not form a gel, but NCs rapidly precipitated upon mixing, forming relatively dense aggregates that stuck to the Eppendorf walls and its bottom.

We used CO oxidation as a model reaction to probe the effective formation of an interface between $\mathrm{Au}$ and $\mathrm{CeO}_{2} \mathrm{NCs}$. Figure 6 shows the catalytic activity of the $\mathrm{Au}-\mathrm{CeO}_{2}$ aerogels toward $\mathrm{CO}$ oxidation compared with those obtained from a $\mathrm{CeO}_{2}$ aerogel. The presence of Au catalytically activates the $\mathrm{CO}$ oxidation at much lower temperature than pure $\mathrm{CeO}_{2}$. The dramatic increase of activity observed for the $\mathrm{Au}-\mathrm{CeO}_{2}$ nanocomposite proves the formation a proper interface between gold and ceria. ${ }^{41,42}$ The optimization of parameters of the catalyst NCs such as size, composition and facets using colloidal synthesis approaches already available will certainly allow maximizing the activity toward this and other reactions.

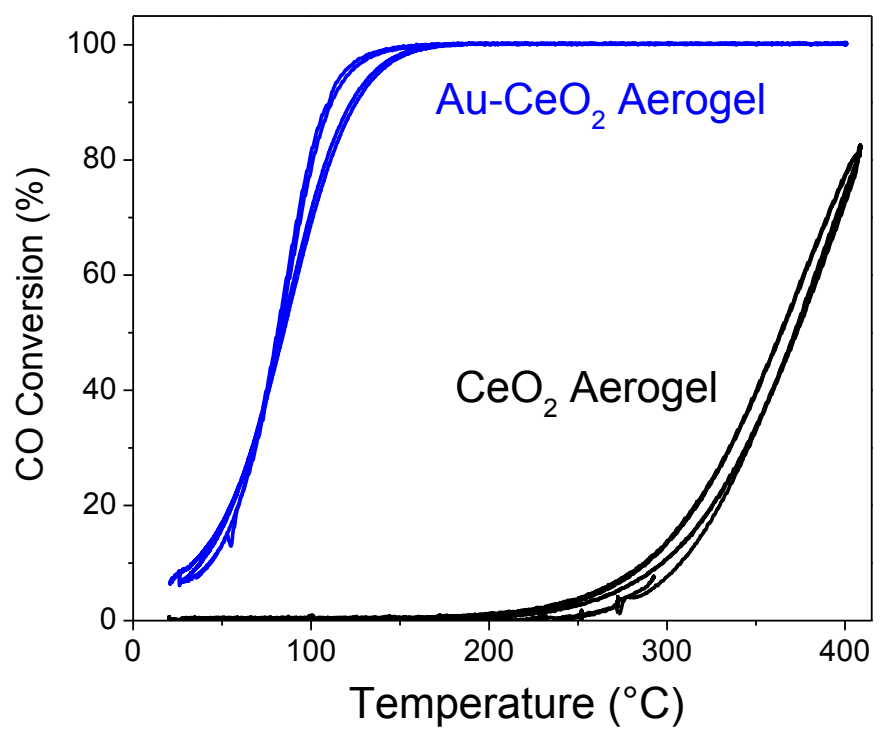


Figure 6. Temperature dependence of the $\mathrm{CO}$ to $\mathrm{CO}_{2}$ conversion on $\mathrm{Au}-\mathrm{CeO}_{2}$ and $\mathrm{CeO}_{2}$ aerogels on two consecutive temperature ramps.

To prove the flexibility of the approach here reported, the same strategy was used to produce gels and aerogels from a combination of a metal chalcogenide and a metal oxide. In this case, the same positively charged NCs were used $\left(\mathrm{Gln}-\mathrm{CeO}_{2}\right)$, while MUA-PbS NCs were selected as the negatively charged NCs. Upon mixing, gelation immediately started and it was completed within $30 \mathrm{~min}$. Following the same procedure as for $\mathrm{Au}-\mathrm{CeO}_{2}$ gels, $\mathrm{PbS}-\mathrm{CeO}_{2}$ gels were dried into aerogels that also showed a highly porous structure. Figure 7 displays a TEM micrograph of the initial $\mathrm{CeO}_{2}$ and PbS NCs, their zeta potential curves, and TEM and SEM images of the gel and aerogel formed from their combination.

As an additional example of the flexibility of the approach here reported, the same strategy was used to produce gels from a combination of $\mathrm{Au}$ and $\mathrm{PbS}$ NCs. In this case, negatively charged MUA-Au NCs were combined with positively-charged PbS NCs obtained by replacing the native organic ligands of PbS NCs with DEAT (see experimental section). Figure S2 displays an optical image of the Au-PbS gel, a TEM micrograph of the xerogel obtained from drying the gel in an ambient atmosphere and the zeta-potential curves of the nanocomposite and the component NCs. 

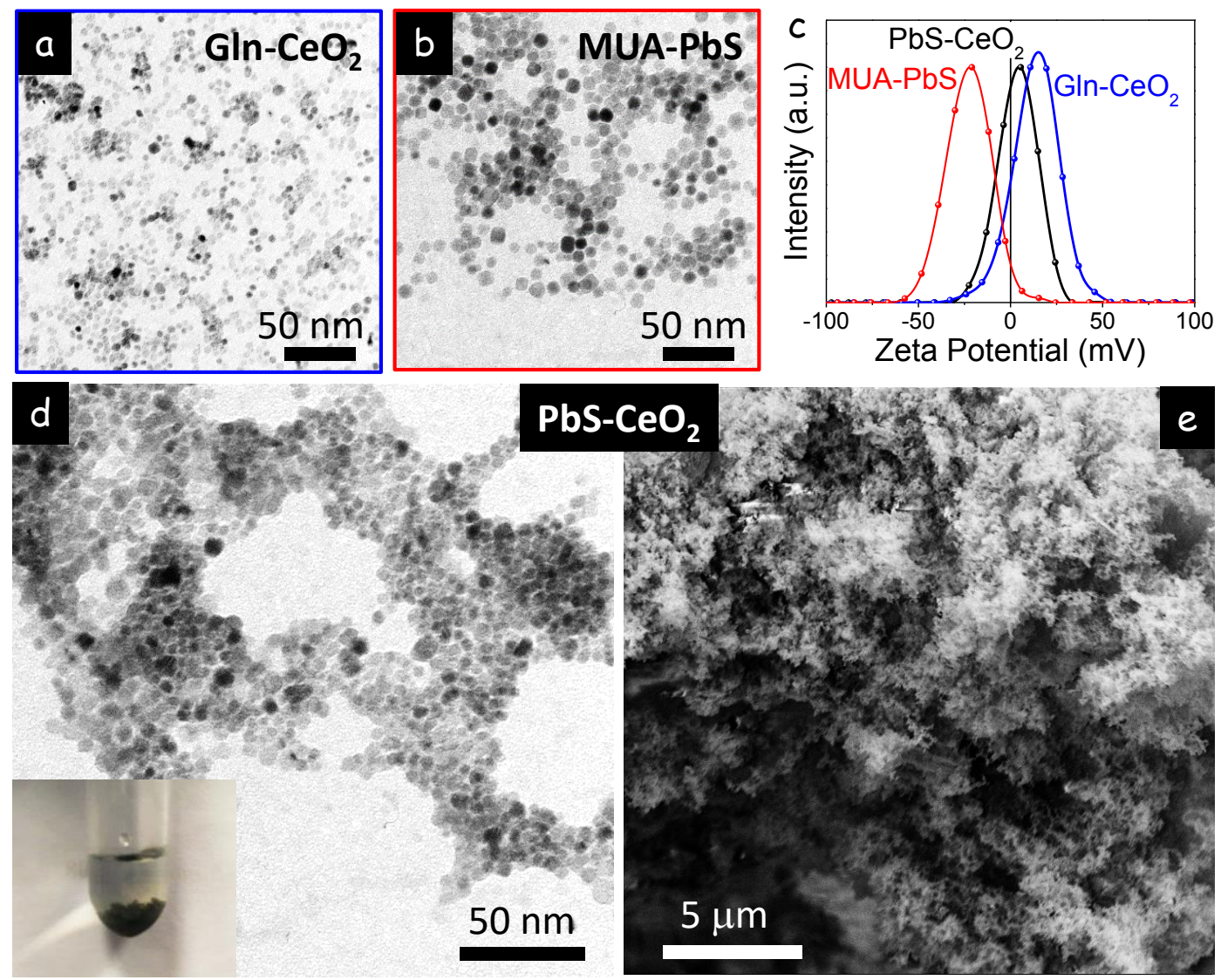

e

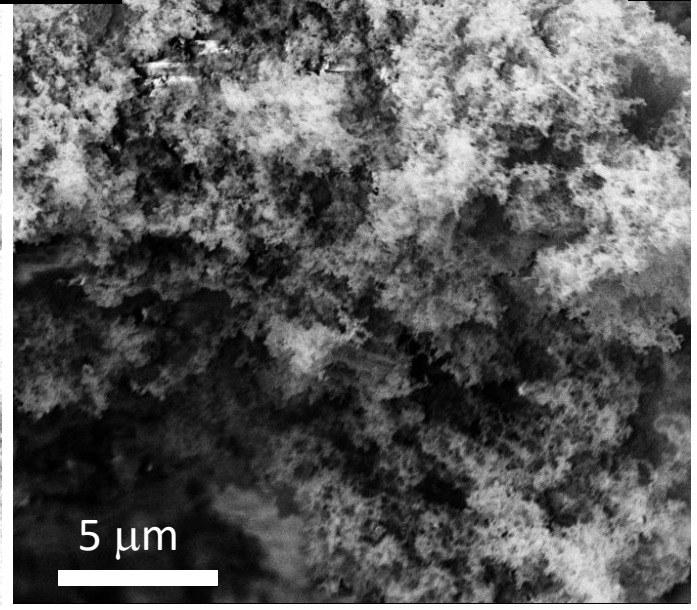

Figure 7. a) TEM image of Gln- $\mathrm{CeO}_{2} \mathrm{NCs}$. b) TEM image of MUA-PbS NCs. c) Zeta potential curves of MUA-PbS, Gln- $\mathrm{CeO}_{2}$ and the gel obtained upon mixing $\mathrm{Gln}-\mathrm{CeO}_{2}$ and $\mathrm{MUA}-\mathrm{PbS} \mathrm{NCs}$.

d) TEM image of the PbS-CeO 2 NC-based gel obtained by mixing $4 \mathrm{ml}$ of a $5 \mathrm{mg} / \mathrm{mL}$ MFA solution of Gln- $\mathrm{CeO}_{2} \mathrm{NCs}$ with $1 \mathrm{ml}$ of a $5 \mathrm{mg} / \mathrm{mL}$ MFA solution of MUA-PbS NCs. e) SEM image of the corresponding $\mathrm{PbS}-\mathrm{CeO}_{2} \mathrm{NC}$-based aerogel.

\section{CONCLUSIONS}

A novel strategy to assemble NCs into multicomponent gels and aerogels based on electrostatic interactions between oppositely charged NCs was presented. Combining positively charged (Glncapped or DEA-capped) NCs with negatively charged (MUA-capped) NCs, metal-metal oxide 
$\left(\mathrm{Au}-\mathrm{CeO}_{2}\right)$, metal chalcogenide-metal oxide $\left(\mathrm{PbS}-\mathrm{CeO}_{2}\right)$ and metal-metal chalcogenide $(\mathrm{Au}-\mathrm{PbS})$ nanocomposites with high specific surface area were produced. The distribution of both NCs was rather homogeneous. We showed that the concentration of NCs in solution and the ratio between the amounts of NCs charged with each charge sign influenced the gel formation and voluminosity. We additionally demonstrated a proper interface between the two compounds, allowing a dramatic increase of the ceria catalytic activity toward $\mathrm{CO}$ oxidation. This new approach can be used to build-up porous nanocomposites with virtually any composition, and allows for a complete control over crystal domain size, facets, crystallographic structure and composition of the nanocomposite porous structures through control of these parameters at the $\mathrm{NC}$ level. This methodology can find numerous applications in fields such as catalysis, chemical sensing, filtering or energy storage to cite just a few.

\section{ASSOCIATED CONTENT}

\section{Supporting Information}

The Supporting Information is available free of charge at DOI:

BJH Pore size distribution of a $\mathrm{Au}-\mathrm{CeO}_{2}$ aerogel. TEM image and zeta potential of the $\mathrm{Au}-\mathrm{PbS}$

NCs gel. Comparison of the catalytic activity of $\mathrm{Au}-\mathrm{CeO}_{2}$ aerogels against similar $\mathrm{Au} @ \mathrm{CeO}_{2}$ systems catalysts reported in literature.

\section{AUTHOR INFORMATION}

\section{Corresponding Authors}

*E-mail: pguardia@irec.cat; acabot@irec.cat

\section{Author Contributions}


The manuscript was written through contributions of all authors. All authors have given approval to the final version of the manuscript.

\section{ACKNOWLEDGMENT}

This work was supported by the European Regional Development Funds and the Spanish MINECO project SEHTOP (ENE2016-77798-C4-3-R) and TEMPTATION (MAT2016-79455P). TB thanks FI-AGAUR Research Fellowship Program, Generalitat de Catalunya (2015 FI_B 00744). PG acknowledges the People Programme (Marie Curie Actions) of the FP7/2007-2013 European Union Program (TECNIOspring grant agreement no. 600388, TECSPR14-1-0053 and TECSPR16-1-0082) and the Agency for Business Competitiveness of the Government of Catalonia, ACCIÓ.

\section{ABBREVIATIONS}

$\mathrm{NC}$, nanocrystal; $\mathrm{CeO}_{2}$, ceria; $\mathrm{Au}$, gold; PbS, lead sulfide; ODE, 1-octadecene; OAm, oleylamine; Gln, L-glutamine; TFA, trifluoroacetic acid; MFA, N-methylformamide; TMAOH, tetramethylammonium hydroxide pentahydrate; TBAB, tert-butylamine-borane complex; tetralin, 1,2,3,4-tetrahydronaphthalene; MUA, 11-mercaptoundecanoic acid; TGA, thioglycolic acid; DEAT, 2-Aminoethanethiol hydrochloride; TEM, transmission electron microscopy; HRTEM, high resolution TEM; SEM, scanning electron microscopy; DLS, dynamic light scattering; FTIR, fourier-transform infrared spectroscopy; SPD, super-critical point dryer; BET, Brunauer-Emmett-Teller; OAm-CeO 2 , OAm-capped $\mathrm{CeO}_{2}$; OAm-Au, OAm-capped Au; OAmPbS, OAm-capped PbS; Gln- $\mathrm{CeO}_{2}$, Gln-capped $\mathrm{CeO}_{2}$; $\mathrm{MUA}-\mathrm{Au}, \mathrm{MUA}$-capped $\mathrm{Au}$; $\mathrm{CO}$, carbon monoxide; MUA-PbS, MUA-capped PbS; CGS, $\mathrm{CuGaS}_{2}$; TGA-CGS, TGA-capped CGS; DEAT-Au, DEAT-capped Au. 


\section{REFERENCES}

(1) Anderson, J. A., Supported metals in catalysis. World Scientific: 2012; 11.

(2) Beloqui Redondo, A.; Ranocchiari, M.; van Bokhoven, J. A., Synthesis of sub-nanometer gold particles on modified silica. Dalton Transactions 2016, 45 (7), 2983-2988.

(3) Gutiérrez, L.-F.; Hamoudi, S.; Belkacemi, K., Synthesis of gold catalysts supported on mesoporous silica materials: recent developments. Catalysts 2011, 1 (1), 97-154.

(4) Li, B.; Hao, Y.; Shao, X.; Tang, H.; Wang, T.; Zhu, J.; Yan, S., Synthesis of hierarchically porous metal oxides and $\mathrm{Au} / \mathrm{TiO}_{2}$ nanohybrids for photodegradation of organic dye and catalytic reduction of 4-nitrophenol. J. Catal. 2015, 329, 368-378.

(5) Rechberger, F.; Niederberger, M., Synthesis of aerogels: from molecular routes to 3dimensional nanoparticle assembly. Nanoscale Horizons 2017, 2 (1), 6-30.

(6) Correa-Baena, J.-P.; Kriz, D. A.; Giotto, M.; Suib, S. L.; Agrios, A. G., Fluoride additive in epoxide-initiated sol-gel synthesis enables thin-film applications of $\mathrm{SnO}_{2}$ aerogels. $R S C$ Advances 2016, 6 (26), 21326-21331.

(7) Laberty-Robert, C.; Long, J. W.; Lucas, E. M.; Pettigrew, K. A.; Stroud, R. M.; Doescher, M. S.; Rolison, D. R., Sol-Gel-Derived Ceria Nanoarchitectures: Synthesis, Characterization, and Electrical Properties. Chem. Mater. 2006, 18 (1), 50-58.

(8) Brinker, C. J.; Scherer, G. W., Sol-Gel Science: the physics and chemistry of sol-gel processing. Elsevier, 1 st ed., 2013.

(9) Mohanan, J. L.; Arachchige, I. U.; Brock, S. L., Porous Semiconductor Chalcogenide Aerogels. Science 2005, 307 (5708), 397-400. 
(10) Ziegler, C.; Wolf, A.; Liu, W.; Herrmann, A.-K.; Gaponik, N.; Eychmüller, A., Modern Inorganic Aerogels. Angew. Chem. Int. Ed. 2017, 56 (43), 13200-13221.

(11) Liu, J.; Meyns, M.; Zhang, T.; Arbiol, J.; Cabot, A.; Shavel, A., Triphenyl phosphite as phosphorous source for the scalable and cost-effective production of transition metal phosphides. Chem. Mater., 2018, 30 (5), 1799-1807.

(12) Liu, Y.; García, G.; Ortega, S.; Cadavid, D.; Palacios, P.; Lu, J.; Ibáñez, M.; Xi, L.; De Roo, J.; López, A. M., Solution-based synthesis and processing of $\mathrm{Sn}$ - and Bi-doped $\mathrm{Cu}_{3} \mathrm{SbSe}_{4}$ nanocrystals, nanomaterials and ring-shaped thermoelectric generators. J. Mater. Chem. A 2017, $5(6), 2592-2602$.

(13) Meyns, M.; Iacono, F.; Palencia, C.; Geweke, J.; Coderch, M. D.; Fittschen, U. E.; Gallego, J. M.; Otero, R.; Juárez, B. H.; Klinke, C., Shape evolution of CdSe nanoparticles controlled by halogen compounds. Chem. Mater. 2014, 26 (5), 1813-1821.

(14) Luo, Z.; Lu, J.; Flox, C.; Nafria, R.; Genç, A.; Arbiol, J.; Llorca, J.; Ibáñez, M.; Morante, J. R.; Cabot, A., Pd 2 Sn [010] nanorods as a highly active and stable ethanol oxidation catalyst. $J$. Mater. Chem. A 2016, 4 (42), 16706-16713.

(15) Yin, Y.; Alivisatos, A. P., Colloidal nanocrystal synthesis and the organic-inorganic interface. Nature 2004, 437 (7059), 664.

(16) Boles, M. A.; Engel, M.; Talapin, D. V., Self-assembly of colloidal nanocrystals: From intricate structures to functional materials. Chem. Rev. 2016, 116 (18), 11220-11289.

(17) Nasilowski, M.; Mahler, B.; Lhuillier, E.; Ithurria, S.; Dubertret, B., Two-Dimensional Colloidal Nanocrystals. Chem. Rev. 2016, 116 (18), 10934-10982. 
(18) Kovalenko, M. V.; Manna, L.; Cabot, A.; Hens, Z.; Talapin, D. V.; Kagan, C. R.; Klimov, V. I.; Rogach, A. L.; Reiss, P.; Milliron, D. J.; Guyot-Sionnnest, P.; Konstantatos, G.; Parak, W. J.; Hyeon, T.; Korgel, B. A.; Murray, C. B.; Heiss, W., Prospects of Nanoscience with Nanocrystals. ACS Nano 2015, 9 (2), 1012-1057.

(19) Li, W.; Zamani, R.; Rivera Gil, P.; Pelaz, B.; Ibáñez, M.; Cadavid, D.; Shavel, A.; AlvarezPuebla, R. A.; Parak, W. J.; Arbiol, J., CuTe nanocrystals: shape and size control, plasmonic properties, and use as SERS probes and photothermal agents. J. Am. Chem. Soc. 2013, 135 (19), 7098-7101.

(20) Berestok, T.; Guardia, P.; Blanco, J.; Nafria, R.; Torruella, P.; López-Conesa, L.; Estradé, S.; Ibáñez, M.; de Roo, J.; Luo, Z.; Cadavid, D.; Martins, J. C.; Kovalenko, M. V.; Peiró, F.; Cabot, A., Tuning Branching in Ceria Nanocrystals. Chem. Mater. 2017, 29 (10), 4418-4424.

(21) Wen, D.; Liu, W.; Haubold, D.; Zhu, C.; Oschatz, M.; Holzschuh, M.; Wolf, A.; Simon, F.; Kaskel, S.; Eychmüller, A., Gold Aerogels: Three-Dimensional Assembly of Nanoparticles and Their Use as Electrocatalytic Interfaces. ACS Nano 2016, 10 (2), 2559-2567.

(22) Liu, W.; Herrmann, A.-K.; Bigall, N. C.; Rodriguez, P.; Wen, D.; Oezaslan, M.; Schmidt, T. J.; Gaponik, N.; Eychmüller, A., Noble Metal Aerogels-Synthesis, Characterization, and Application as Electrocatalysts. Acc. Chem. Res. 2015, 48 (2), 154-162.

(23) Hendel, T.; Lesnyak, V.; Kühn, L.; Herrmann, A. K.; Bigall, N. C.; Borchardt, L.; Kaskel, S.; Gaponik, N.; Eychmüller, A., Mixed Aerogels from Au and CdTe Nanoparticles. Adv. Funct. Mater. 2013, 23 (15), 1903-1911. 
(24) Voitekhovich, S. V.; Lesnyak, V.; Gaponik, N.; Eychmüller, A., Tetrazoles: Unique Capping Ligands and Precursors for Nanostructured Materials. Small 2015, 11 (43), 5728-5739.

(25) Heiligtag, F. J.; Rossell, M. D.; Suess, M. J.; Niederberger, M., Template-free co-assembly of preformed $\mathrm{Au}$ and $\mathrm{TiO}_{2}$ nanoparticles into multicomponent 3D aerogels. J. Mater. Chem. 2011, 21 (42), 16893-16899.

(26) Da Silva, R. O.; Heiligtag, F. J.; Karnahl, M.; Junge, H.; Niederberger, M.; Wohlrab, S., Design of multicomponent aerogels and their performance in photocatalytic hydrogen production. Catal. Today 2015, 246, 101-107.

(27) Peng, S.; Lee, Y.; Wang, C.; Yin, H.; Dai, S.; Sun, S., A facile synthesis of monodisperse Au nanoparticles and their catalysis of CO oxidation. Nano Research 2008, 1 (3), 229-234.

(28) Ibáñez, M.; Korkosz, R. J.; Luo, Z.; Riba, P.; Cadavid, D.; Ortega, S.; Cabot, A.; Kanatzidis, M. G., Electron Doping in Bottom-Up Engineered Thermoelectric Nanomaterials through HClMediated Ligand Displacement. J. Am. Chem. Soc. 2015, 137 (12), 4046-4049.

(29) De Roo, J.; Coucke, S.; Rijckaert, H.; De Keukeleere, K.; Sinnaeve, D.; Hens, Z.; Martins, J. C.; Van Driessche, I., Amino Acid-Based Stabilization of Oxide Nanocrystals in Polar Media: From Insight in Ligand Exchange to Solution ${ }^{1} \mathrm{H}$ NMR Probing of Short-Chained Adsorbates. Langmuir 2016, 32 (8), 1962-1970.

(30) Etgar, L.; Lifshitz, E.; Tannenbaum, R. Synthesis of water-soluble PbSe quantum dots. J. Mater. Res. 2008, 23 (4), 899-903. 
(31) Dragoman, R. M.; Grogg, M.; Bodnarchuk, M. I.; Tiefenboeck, P.; Hilvert, D.; Dirin, D. N.; Kovalenko, M. V., Surface-Engineered Cationic Nanocrystals Stable in Biological Buffers and High Ionic Strength Solutions. Chem.Mater. 2017, 29 (21), 9416-9428.

(32) Kalsin, A. M.; Fialkowski, M.; Paszewski, M.; Smoukov, S. K.; Bishop, K. J.; Grzybowski, B. A., Electrostatic self-assembly of binary nanoparticle crystals with a diamond-like lattice. Science 2006, 312 (5772), 420-424.

(33) Gittins, D. I.; Caruso, F., Spontaneous phase transfer of nanoparticulate metals from organic to aqueous media. Angew. Chem. Int. Ed. 2001, 40 (16), 3001-3004.

(34) Sapsford, K. E.; Algar, W. R.; Berti, L.; Gemmill, K. B.; Casey, B. J.; Oh, E.; Stewart, M. H.; Medintz, I. L., Functionalizing Nanoparticles with Biological Molecules: Developing Chemistries that Facilitate Nanotechnology. Chem. Rev. 2013, 113 (3), 1904-2074.

(35) Meador, M. A. B.; Fabrizio, E. F.; Ilhan, F.; Dass, A.; Zhang, G.; Vassilaras, P.; Johnston, J. C.; Leventis, N., Cross-linking Amine-Modified Silica Aerogels with Epoxies: Mechanically Strong Lightweight Porous Materials. Chem. Mater. 2005, 17 (5), 1085-1098.

(36) Leopold, L. F.; Tódor, I. S.; Diaconeasa, Z.; Rugină, D.; Ştefancu, A.; Leopold, N.; Coman, C., Assessment of PEG and BSA-PEG gold nanoparticles cellular interaction. Colloids Surf. A 2017, 532, 70-76.

(37) Kimura, K.; Takashima, S.; Ohshima, H., Molecular Approach to the Surface Potential Estimate of Thiolate-Modified Gold Nanoparticles. J. Phys. Chem. B 2002, 106 (29), 7260-7266. 
(38) Laaksonen, T.; Ahonen, P.; Johans, C.; Kontturi, K., Stability and Electrostatics of Mercaptoundecanoic Acid-Capped Gold Nanoparticles with Varying Counterion Size. ChemPhysChem 2006, 7 (10), 2143-2149.

(39) Amoli, B. M.; Gumfekar, S.; Hu, A.; Zhou, Y. N.; Zhao, B., Thiocarboxylate functionalization of silver nanoparticles: effect of chain length on the electrical conductivity of nanoparticles and their polymer composites. J. Mater. Chem. 2012, 22 (37), 20048-20056.

(40) Chen, S.; Kimura, K., Synthesis and Characterization of Carboxylate-Modified Gold Nanoparticle Powders Dispersible in Water. Langmuir 1999, 15(4), 1075-1082.

(41) Trovarelli, A.; Llorca, J., Ceria Catalysts at Nanoscale: How Do Crystal Shapes Shape Catalysis? ACS Catalysis 2017, 7 (7), 4716-4735.

(42) Wu, C.; Dang, Z.; Prato, M.; Marras, S.; Cerea, A.; De Angelis, F.; Manna, L.; Colombo, M. Nanosized, Hollow, and Mn-Doped $\mathrm{CeO}_{2} / \mathrm{SiO}_{2}$ Catalysts via Galvanic Replacement: Preparation, Characterization, and Application as Highly Active Catalysts. ACS Appl. Nano Mater. 2018, 1 (4), 1438-1443. 
TOC
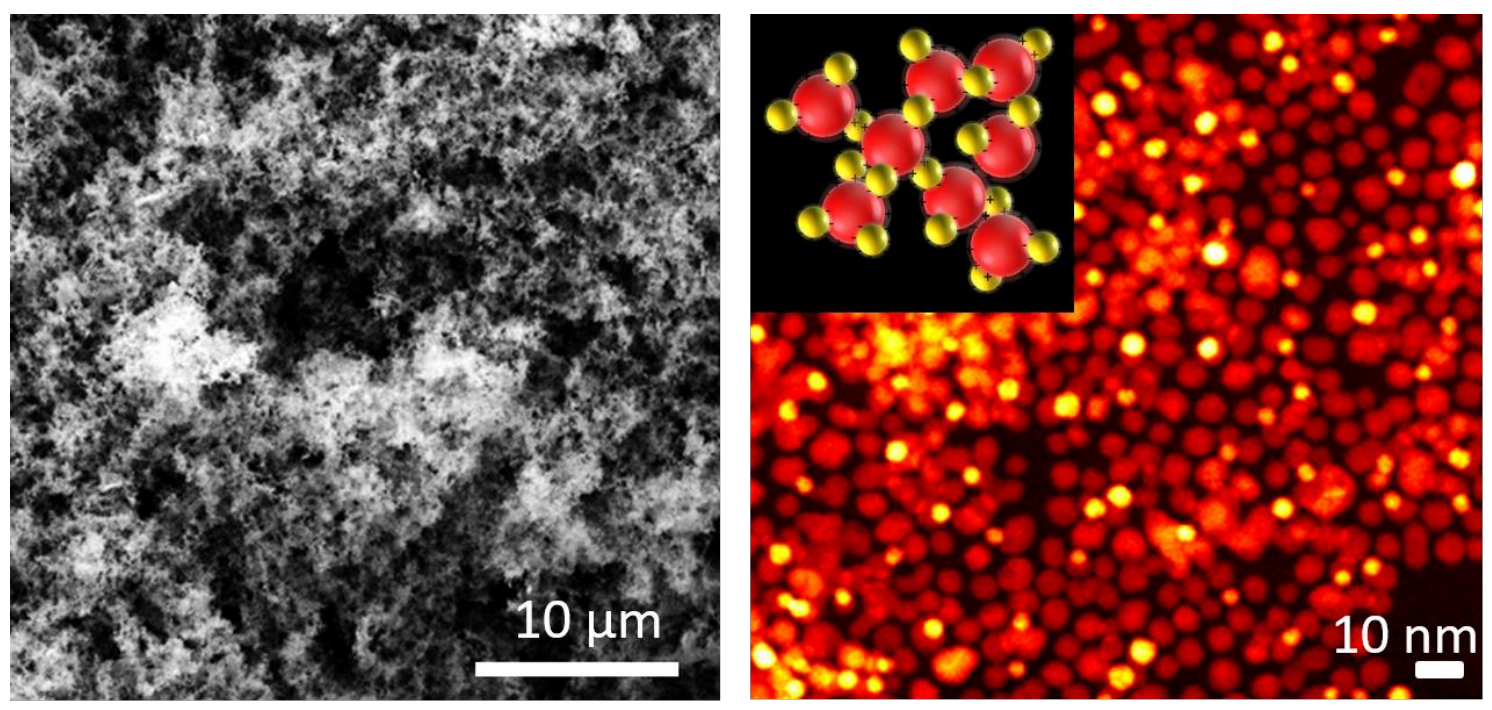